\title{
Research and Exploration on the Bachelor Thesis Topic Selection and Guidance of Undergraduate Students
}

\author{
DU Dongxing \\ College of Electromechanical Engineering, Qingdao University of Science and Technology, \\ Qingdao 266061, China
}

Email: du-dongxing@163.com

\begin{abstract}
Keywords: Bachelor thesis, topic selection, guidance, research, exploration
Abstract. Combined with the practical experiences in the year of 2009-2015, we carried out studies on the aspects of bachelor thesis topic selection and guidance of undergraduate students in the specialty of Engineering Thermophysics in the Department of Energy and Power Engineering in our university. This paper suggests that the topic should be closely related to the professional disciplines and focus on the specialty priority development fields, and the effective guidance of the students has the same importance as the topic selection for a satisfactory bachelor thesis. Study results indicate good thesis topics and high effective guidance are of great significance to the undergraduate students and as well to the guidance teacher.
\end{abstract}

\section{Introduction}

Engineering thermophysics is a study of the laws of the transformation of energy in the form of heat and its applications. It studies the internal laws of various kinds of thermal phenomena and thermal processes, and it is used to guide the engineering practice. Engineering thermal physics has its own basic laws: the first law and the second law of thermodynamics, the law of Newton's mechanics, the law of heat and mass transfer and the law of chemical kinetics. Engineering thermophysics is a complete system of science and technology, including not only knowledge innovation, but also technological innovation ${ }^{[1]}$.

Engineering thermophysics is the main fundamental subject in the area of energy usage, and its development promotes the progress of energy technology. Energy related problems, such as energy development, environmental protection, energy saving and emission reduction, are the long-term constraint and bottleneck of social and economic development, therefore they are always the major issues to ensure the supply of clean, economic, adequate and safe energy. Facing on the call of the times, the development of engineering thermophysics is not only to adapt to the characteristics of the dispersed renewable energy sources, but also to be capable to provide energy for the development of large industries. In this process, the subject of engineering thermophysics will face new opportunities and challenges, and its developments will have a significant impact on the human society. ${ }^{[2}$

At present, our university owns doctorate authorization in the first-level discipline of power engineering and engineering thermophysics. In recent years, teachers in this discipline have undertaken numbers of national, provincial and municipal scientific research projects, and have received numbers of scientific and technological awards. As one of the six second-level disciplines, the thermophysics subject in our university has following research contents: (1) heat transfer enhancement; (2) thermal physical properties of materials; (3) new heat transfer technologies and equipments; (4) simulation and optimization of thermal systems; (5) numerical simulation and engineering application. ${ }^{[3]}$

Benifiting from advanced discipline system, competitive teaching staff, rigorous academic atmosphere and the strongly support from the univisity, the professional level of the engineering thermophysics discipline has been greatly improved, in the mean time the training of undergraduate students has also achieved remarkable achievements. However, undergraduate thesis still has some room for improvement, especially in the current employment environment. Originally, the time for undergraduate thesis is quite tight, and many students need to spend extra amounts of time and efforts to pass the entrance exam to become a postgraduate or to find a job, therefore the students are 
commonly lack of passion for the bachelor thesis work, and consequently their graduation theses usually contain little helpful information for promoting the teaching and researching works of the university. Based on the current situation and seven years of working practice, the author studies and explores the ways to imporve undergraduate thesis from the aspects of thesis topic selection and daily guidance in the thesis progress.

\section{The guiding ideology for topic selection of the undergraduate thesis}

The topic of the thesis should follow the discipline development direction and priority development field, which is the prerequisite to improve the level of undergraduate thesis. At present, there are five professional directions and four priority development areas in the specialty of engineering thermophysics. Five professional directions are engineering thermodynamics and energy utilization, thermal machine gas dynamic thermodynamics and fluid machinery, heat and mass transfer, combustion science and multiphase flow sub disciplines. While the four priority areas of development are energy conservation and scientific usage of energy, coal clean utilization technology, vigorous development of renewable energy, and greenhouse gas control strategy for $\mathrm{CO} 2$ capture and sequestration.

The topic of undergraduate graduation thesis should be based on the academic background of teachers, the discipline advantages of the university, and be closely linked to the development directions and priority development areas of the subject. As for multiphase flow sub-discipline, teachers should understand the current research focus on the two-phase flow mathematical models and numerical simulation methods, and the study on three-phase flow, although still in the initial stage, will gradually become the focus of the research. The numerical simulation methods show new ideas and prospects in the aspects of gas (vapor)/liquid and liquid/liquid interface, gas/solid and liquid/solid multiphase flow, gas/liquid/solid three phase flow, and so on. In addition, extensive researches have also been carried out on the test methods of multiphase flow and transfer parameters. While on the priority development direction of the greenhouse gas control strategy and $\mathrm{CO} 2$ capture and sequestration, teachers should know the recent main measures to control greenhouse gas emissions is to develop energy efficiency enhancement and resource utilization technologies, the middle-term focus is to develop renewable energy and other alternative energy sources, while the long-term strategy is to develop efficient $\mathrm{CO} 2$ capture and storage technologies. Because present $\mathrm{CO} 2$ capture and sequestration (CCS) technology is still unable to meet the requirements of sustainable development, the "revolutionary" technology suitable for Chinese conditions, should be suggested to achieve the goals of high energy utilization efficiency and low cost $\mathrm{CO} 2$ emission reduction.

Based on the profound understanding of the discipline, the teacher can determine the research direction and guiding ideology of the thesis. As in the author's case, the thesis topics are determined to be the multiphase flow in porous media aiming for $\mathrm{CO} 2$ foam application in Enhanced Oil Recovery (EOR). It is also expected that $\mathrm{CO} 2$ involved in the oil displacement process can be buried in the ground, thereby can effectively reduce the emission of the greenhouse gas to achieve sustainable development of human society. Therefore, the following topics are selected in the years of 2009-2015.

A typical topic for bachelor thesis in 2009 is: "carbon dioxide foam flow characteristics in the tube". The typical topics in 2010 are: "effect of surfactant on the surface tension", "model analysis for film foam flow characteristics in a tube" and "the effect of internal gas phase on bubble film flow characteristics". The typical topics in 2011 are: "visualization research on bubble flow process" and "study on air film foam rheology in a tube ". The typical topics in 2012 are: " dynamic simulation of flowing film bubble in a straight tube"," non-dimensional analysis for bubble film flow in tubes"," film foam flow characteristics in a periodic converging-diverging tube " and " $\mathrm{CO} 2$ foam seepage characteristics in porous media". The typical topics in 2013 are: " design and production of data acquisition system for bubble flow rheology", "measurement of homogeneous porous medium characteristic parameters and repetitive analysis of the production" ,"measurement of heterogeneous porous medium characteristic parameters", "theoretical analysis for film foam flow characteristics in a 
converging-diverging tube" etc. The typical topics in 2014 are: "experimental study on air foam flow in a heterogeneous porous media", "numerical simulation for foam flow characteristics in porous media", "LBM study on fluid flow around a circular tube", etc. The typical topics in 2015 include "digital processing of core samples and characteristic parameters analysis", "CO2- water-oil system phase equilibrium analysis", "numerical simulation for foam flow in porous media based on bubble population balance model", etc. All the topics are focus on multiphase foam technology in energy exploration and greenhouse gas sequestration, which is the priority development direction of the present engineering thermophysics subject, and are all locate in the interdisciplinary research field involving chemistry, chemical engineering, physics and mechanics, with certain academic level and practical background.

\section{The guidance of undergraduate students}

Although the set time for undergraduate graduation thesis is in the eighth semester, the guidance teacher should select and determine candidates before the end of the seventh semester. Take 8 undergraduate students as an example, teachers should meet and talk to each candidate to learn their academic interests and backgrounds, and then form 3-4 research groups with different themes such as experiments, calculations, image processing and so on. Each candidate should get his (or her) specific topic and the corresponding references, which he (or she) needs to read in the winter holiday period.

Within one month after the start of the eighth semester, the instructor should discuss with one group every week, so as to ensure that each student has more than 30 minutes of academic discussion time when the teacher can explain in depth the subject to establish the student's confidence to complete the graduation thesis. In the second month of eighth semester, teacher should maintain the academic meeting frequency of one month to help students pass the mid-term thesis evaluation, without sacrificing the precious time which the students may use to take part in the entrance oral interview for postgraduate study or to find a good job. In the third month of the eighth semester, the teacher should increase the supervision and guidance strength to twice face-to-face 1-hour discussion meetings each month with each undergraduate student. The third month is essential, for most fruitful works, such as a certain amount of experimental data, well debugged programs or reasonable analytical values, should be obtained in this period of time. In the fourth month, which is the last month for students to finish and defense their theses, the teacher should mainly focus on reading the students' reports and analyzing the results. If the teacher found some interesting or indicative experimental or numerical phenomena, the teacher could guide the students for further study and to encourage the students to complete their undergraduate theses in a higher standard.

In summary, the instructor should grasp the time node in the guidance process of undergraduate student. Besides, the teacher has to guide the students by himself instead of putting them under the supervision of postgraduate students. The teacher also needs to guide the students according to their different personal characteristics to ultimately achieve the purpose of common development.

\section{The significance of undergraduate thesis to teachers and undergraduates}

Good thesis topics are generally aligned to the development direction of the discipline and own wide application potentials, therefore they are likely to stimulate the enthusiasm of the teacher and the students. In addition, the responsible guidance of teachers can significantly enhance the academic interactions between teachers and students, and further improve the level of teachers' scientific research and undergraduates' ability on mastering and usage of the taught knowledge. By employing the described principles on topic selection and student guidance, the teacher could obtain substantial results and publish high level papers. As in author's case, the teacher together with instructed undergraduates has published dozens of high level papers, among which 3 are indexed by SCI and 8 are indexed by EI or ISTP.

Of course, a good topic of the thesis could put forward higher requirements for both teachers and undergraduate students. To meet the requirements, the teacher should always walk in the forefront of the subject, often refer to up-to-date literatures and participate in the high level academic conferences 
in order to maintain clear understanding on recent developments and tendencies of the subject. While students should work dependably and always keep the academic communication with the teacher, and strive to cultivate the passion for scientific research and practical works.

\section{Concluding remarks}

Research and exploration have been carried out on the aspects of bachelor thesis topic selection and guidance principles of undergraduate students based on author's practical experiences in the year of 2009-2015. The paper suggests that the thesis topic should be closely related to discipline development directions and focus on the priority areas of the subject, so as to improve the positioning of undergraduate thesis work. The strategic guidance has the same importance as the topic selection for obtaining a satisfactory bachelor thesis. Through the bachelor thesis segment, the teacher can cultivate the innovative spirit of the graduates by distilling their four years professional knowledge from the practical research works. A properly progressed undergraduate thesis will not only improve the professional self-confidence of college graduates but also help teachers for their scientific or application researches. It is indicated that good thesis topics and effective guidance are of great significance to the undergraduate students and as well to the guidance teachers themselves.

\section{Acknowledgements}

This bachelor theses with topics listed in this paper are finished under the support of the Natural Science Foundation of China (51476081).

\section{References}

[1] Research Report on Development of the Discipline of Engineering Thermophysics (Simple version). http://wenku.baidu.com/view/4ee49818650e52ea551898af.html (in Chinese)

[2] Research Report on Development Strategy of the Discipline of Engineering Thermophysics and Energy Utilization (2011-2020). Edited by the engineering and material Science Department of The National Natural Science Fund Committee, China Science Publication \& Media Ltd. (CSPM), 2011.11 (in Chinese)

[3] Introduction for the first level discipline of power engineering and thermophysics in QUST, http://www.100exam.com/WebSpecF/EnrolDetail.aspx?id=70528 (in Chinese) 